\title{
Enhanced Quadrupole and Octupole Strength in Doubly Magic ${ }^{132} \mathrm{Sn}$
}

D. Rosiak, ${ }^{1}$ M. Seidlitz, ${ }^{1, *}$ P. Reiter, ${ }^{1}$ H. Naïdja ${ }^{2,3,4}$ Y. Tsunoda, ${ }^{5}$ T. Togashi, ${ }^{5}$ F. Nowacki,${ }^{2,3}$ T. Otsuka,,${ }^{6,5,7,9}$ G. Colò, ${ }^{10,11}$ K. Arnswald, ${ }^{1}$ T. Berry, ${ }^{12}$ A. Blazhev, ${ }^{1}$ M. J. G. Borge, ${ }^{13, \dagger}$ J. Cederkälll, ${ }^{14}$ D. M. Cox,${ }^{15,16}$ H. De Witte, ${ }^{8}$ L. P. Gaffney, ${ }^{13}$ C. Henrich, ${ }^{17}$ R. Hirsch, ${ }^{1}$ M. Huyse, ${ }^{8}$ A. Illana, ${ }^{8}$ K. Johnston, ${ }^{13}$ L. Kaya, ${ }_{1}^{1}$ Th. Kröll, ${ }^{17}$ M. L. Lozano Benito, ${ }^{13}$ J. Ojala, ${ }^{15,16}$ J. Pakarinen, ${ }^{15,16}$ M. Queiser, ${ }^{1}$ G. Rainovski, ${ }^{18}$ J. A. Rodriguez, ${ }^{13}$ B. Siebeck, ${ }^{1}$ E. Siesling, ${ }^{13}$ J. Snäll, ${ }^{14}$ P. Van Duppen, ${ }^{8}$ A. Vogt, ${ }^{1}$ M. von Schmid, ${ }^{17}$ N. Warr, ${ }^{1}$ F. Wenander, ${ }^{13}$ and K. O. Zell ${ }^{1}$

(MINIBALL and HIE-ISOLDE Collaborations)

${ }^{1}$ Institut für Kernphysik, Universität zu Köln, Zülpicher Straße 77, D-50937 Köln, Germany

${ }^{2}$ Université de Strasbourg, IPHC, 23 rue du Loess, F-67037 Strasbourg, France

${ }^{3}$ CNRS, UMR 7178, F-67037 Strasbourg, France

${ }^{4}$ Université Constantine 1, LPMS, route Ain El Bey, DZ-25000 Constantine, Algeria

${ }^{5}$ Center for Nuclear Study, University of Tokyo, Hongo, Bunkyo-ku, Tokyo 113-0033, Japan

${ }^{6}$ Department of Physics, University of Tokyo, Hongo, Bunkyo-ku, Tokyo 113-0033, Japan

${ }^{7}$ RIKEN Nishina Center, 2-1 Hirosawa, Wako, Saitama 351-0198, Japan

${ }^{8}$ Instituut voor Kern- en Stralingsfysica, KU Leuven, Celestijnenlaan 200D, B-3001 Leuven, Belgium

${ }^{9}$ National Superconducting Cyclotron Laboratory, Michigan State University, East Lansing, Michigan 48824, USA

${ }^{10}$ Dipartimento di Fisica, Universitò degli Studi di Milano, Via Celoria 16, I-20133 Milano, Italy

${ }^{11}$ INFN sezione di Milano, Via Celoria 16, I-20133, Milano, Italy

${ }^{12}$ Department of Physics, University of Surrey, Guildford, GU2 7XH, United Kingdom

${ }^{13}$ ISOLDE, CERN, CH-1211 Geneva 23, Switzerland

${ }^{14}$ Department of Physics, Lund University, Box 118, SE-221 00 Lund, Sweden

${ }^{15}$ University of Jyvaskyla, Department of Physics, P. O. Box 35, FI-40014 University of Jyvaskyla, Finland

${ }^{16}$ Helsinki Institute of Physics, P. O. Box 64, FI-00014 Helsinki, Finland

${ }^{17}$ Institut für Kernphysik, Technische Universität Darmstadt, Schlossgartenstraße 9, D-64289 Darmstadt, Germany

${ }^{18}$ Department of Atomic Physics, University of Sofia, 5 James Bourchier Boulevard, BG-1164 Sofia, Bulgaria

(Received 28 May 2018; revised manuscript received 14 September 2018; published 18 December 2018)

The first $2^{+}$and $3^{-}$states of the doubly magic nucleus ${ }^{132} \mathrm{Sn}$ are populated via safe Coulomb excitation employing the recently commissioned HIE-ISOLDE accelerator at CERN in conjunction with the highly efficient MINIBALL array. The ${ }^{132} \mathrm{Sn}$ ions are accelerated to an energy of $5.49 \mathrm{MeV} /$ nucleon and impinged on a ${ }^{206} \mathrm{~Pb}$ target. Deexciting $\gamma$ rays from the low-lying excited states of the target and the projectile are recorded in coincidence with scattered particles. The reduced transition strengths are determined for the transitions $0_{\mathrm{g} . \mathrm{s} .}^{+} \rightarrow 2_{1}^{+}, 0_{\mathrm{g} . \mathrm{s} .}^{+} \rightarrow 3_{1}^{-}$, and $2_{1}^{+} \rightarrow 3_{1}^{-}$in ${ }^{132} \mathrm{Sn}$. The results on these states provide crucial information on cross-shell configurations which are determined within large-scale shellmodel and Monte Carlo shell-model calculations as well as from random-phase approximation and relativistic random-phase approximation. The locally enhanced $B\left(E 2 ; 0_{\mathrm{g} . \mathrm{s} .}^{+} \rightarrow 2_{1}^{+}\right)$strength is consistent with the microscopic description of the structure of the respective states within all theoretical approaches. The presented results of experiment and theory can be considered to be the first direct verification of the sphericity and double magicity of ${ }^{132} \mathrm{Sn}$.

DOI: 10.1103/PhysRevLett.121.252501

Ten doubly magic atomic nuclei act as cornerstones along the whole chart of nuclei. Their basic properties like masses, binding energies, and excited states play an

Published by the American Physical Society under the terms of the Creative Commons Attribution 4.0 International license. Further distribution of this work must maintain attribution to the author(s) and the published article's title, journal citation, and DOI. eminent role for a detailed understanding and theoretical description of the nuclear system. Tin has a magic number of protons $(Z=50)$ and is the heaviest element to have two isotopes with a magic number of neutrons $\left({ }^{100} \mathrm{Sn}_{50}\right.$ and ${ }^{132} \mathrm{Sn}_{82}$ ). The latter of these nuclei acts as an essential benchmark for theoretical approaches extending towards heavier and more neutron-rich systems. This region of the nuclear chart plays a critical role in the astrophysical $r$ process, and understanding its path around ${ }^{132} \mathrm{Sn}$ is 
indispensable for the accurate description of elemental abundances in the Solar System [1]. Recent multimessenger astronomy of, e.g., kilonovae reported on observation of nucleosynthesis of medium-heavy and heavy neutronrich nuclei around $N=50$ and $N=82$ [2,3]. The persistence or disappearance of the $N=82$ shell in neutron-rich nuclei implies severe consequences for modeling the $r$-process nucleosynthesis.

While the single-particle states of neighboring isotopes were studied in pioneering experiments recently [4-9], the complementary collective properties of ${ }^{132} \mathrm{Sn}$ are poorly understood, and experimental information on low-lying excitations is severely lacking. In particular, the collective behavior of nucleons giving rise to the simplest vibrational modes of quadrupole and octupole character is dependent on shell energies and effective interactions; thus, measuring these properties acts as a sensitive probe of shell-model (SM) calculations. Especially important are the constraints these properties provide on the extrapolation of theoretical models to neutron-rich nuclei. While nuclei around $Z=50, N=82$ could be treated within the SM, predictions for doubly magic ${ }^{132} \mathrm{Sn}$, its excited $2_{1}^{+}$and $33_{1}^{-}$states, and the respective electromagnetic properties were not accessible until now due to the huge valence space and computational complexity including several shells for protons and neutrons simultaneously. Models based on the (quasiparticle) random-phase approximation (RPA) have, however, been used for the description of tin isotopes, predicting a local increase of the quadrupole and octupole collectivity at ${ }^{132} \mathrm{Sn}$ [10-12]. This enhancement is explained by the excitation of a doubly magic core and the occupation of specific orbitals across a large shell gap and is a crucial feature of doubly magic nuclei. A similar behavior is observed along the lead isotopic chain $[11,12]$ and is also expected for so far unreachable ${ }^{78} \mathrm{Ni}[13,14]$.

The huge experimental and theoretical interest in ${ }^{132} \mathrm{Sn}$ and its vicinity goes along with advanced detection techniques and new facilities for reaccelerated radioactive ion beams. The first preliminary results on Coulomb excitation of ${ }^{130,132,134} \mathrm{Sn}$ were obtained at HRIBF and published in conference proceedings [15-18]. The present Letter reports the results for the $2_{1}^{+}$and $33_{1}^{-}$states in ${ }^{132} \mathrm{Sn}$ and their transition strengths obtained in a Coulombexcitation (CE) experiment performed at the newly commissioned HIE-ISOLDE facility at CERN $[19,20]$. Highresolution gamma-ray spectroscopy is employed for the unambiguous identification of the interesting transitions in ${ }^{132} \mathrm{Sn}$ in contrast to the former measurements.

The radioactive ${ }^{132} \mathrm{Sn}$ was produced by irradiating a thick $\mathrm{UC}_{x}$ target with $1.4-\mathrm{GeV}$ protons provided by the CERN PS Booster. The average current of the pulsed proton beam amounted to $2(\mu) \mathrm{A}$. To reduce intense isobaric contamination, the isotope of interest ${ }^{132} \mathrm{Sn}$ was extracted as a positively charged $\left({ }^{132} \mathrm{Sn}^{34} \mathrm{~S}\right)^{1+}$ molecular beam (cf. Ref. [21]), which was then mass separated by the
ISOLDE high-resolution separator (HRS). The molecular beam with $A=166$ was guided into a Penning trap (REXTRAP) [22], where it was accumulated, cooled, and bunched. Following break up of the molecules by charge exchange, the residual ${ }^{132} \mathrm{Sn}^{1+}$ ions were injected into an electron-beam ion source (REXEBIS) [23] where they were charge bred for $194 \mathrm{~ms}$. After a mass-overcharge separation with $A / q=4.258$, the ${ }^{132} \mathrm{Sn}^{31+}$ beam was postaccelerated by the HIE-ISOLDE linear accelerator [20] and delivered with a final beam energy of $5.49 \mathrm{MeV} /$ nucleon onto the secondary target inside the MINIBALL array [24]. The secondary target with a thickness of $3.1 \mathrm{mg} / \mathrm{cm}^{2}$ consisted of $99.80 \%$-enriched ${ }^{206} \mathrm{~Pb}$ evaporated onto a $25-\mu \mathrm{g} / \mathrm{cm}^{2}$ thin ${ }^{12} \mathrm{C}$ supporting foil. The average intensity of the postaccelerated ion beam amounted to approximately $3.0 \times 10^{5}$ ions/s, with an overall beamon-target time of $127 \mathrm{~h}$. Scattered beam and target nuclei were detected by a highly segmented circular double-sided silicon strip detector (DSSSD), covering scattering angles between $16.2^{\circ}$ and $52.9^{\circ}$ in the laboratory system $[24,25] . \gamma$ rays following $\mathrm{CE}$ of projectile and target nuclei were detected in coincidence by the MINIBALL $\gamma$-ray spectrometer, consisting of eight triple-cluster detectors in close geometry, each containing three sixfold-segmented HPGe crystals $[24,26]$.

Accurate energy and efficiency calibrations of the HPGe detectors up to $5 \mathrm{MeV}$ were achieved using ${ }^{60} \mathrm{Co},{ }^{152} \mathrm{Eu}$, and ${ }^{66} \mathrm{Ga}$ sources yielding a $\gamma$-ray efficiency of $2.74(4) \%$ at $4 \mathrm{MeV}$. The position of each individual segment of the HPGe detectors was determined utilizing Doppler-shifted $\gamma$ rays of the reactions $d\left({ }^{22} \mathrm{Ne},{ }^{23} \mathrm{Ne}\right) p$ and $d\left({ }^{22} \mathrm{Ne},{ }^{23} \mathrm{Ne}\right) n$. Combining the angular information of the $\gamma$ rays with the momentum vector of the scattered nucleus that was detected in the DSSSD in coincidence, the final relative energy resolution of the $\gamma$-ray transitions from $\mathrm{CE}$ amounted to $1.1 \%$ (FWHM) after Doppler correction. This ensures a clean separation and identification of different $\gamma$-ray transitions of ${ }^{132} \mathrm{Sn}$ even at energies above $4 \mathrm{MeV}$.

The exact beam composition was determined using $\beta$-delayed $\gamma$-ray events as well as prompt $\gamma$-ray events after CE. No isobaric contaminations stemming from ${ }^{132} \mathrm{Cs}$ and ${ }^{132}$ In were observed by studying $\beta$ decays. Moreover, timedependent intensity ratios of decays of mother and daughter nuclei in the decay chain of ${ }^{132} \mathrm{Sn}$ during the beam time showed no evidence for ${ }^{132} \mathrm{I}$ in the beam. $\gamma$-ray spectra obtained from prompt $\mathrm{CE}$ events were particularly clean from ${ }^{132} \mathrm{Te}$ and ${ }^{132} \mathrm{Xe}$ [cf. Fig. 1(a)]. Considering their known transition probabilities [27], both isotopes were excluded from being beam contaminants. The contribution of ${ }^{132} \mathrm{Ba}$ to the beam composition was determined from the measured $2_{1}^{+} \rightarrow 0_{\mathrm{g} . s .}^{+}$transition at $464 \mathrm{keV}$ and its known $B(E 2)$ value [27]. The amount of ${ }^{132} \mathrm{Sb}$ in the beam was determined relative to the yield of ${ }^{132} \mathrm{Sn}$ via a dedicated implantation and subsequent decay measurement at the 
target position inside the MINIBALL setup. The calculated ratio amounted to $I\left({ }^{132} \mathrm{Sb}\right) / I\left({ }^{132} \mathrm{Sn}\right)=0.16(1)$. As the mass number of ${ }^{166} \mathrm{Yb}$ equals the mass number of the extracted molecular ${ }^{132} \mathrm{Sn}^{34} \mathrm{~S}$ beam, both ion species were transported through the HRS into the REXTRAP and the REXEBIS. Moreover, a similar $A / q$ ratio of 4.256 for ${ }^{166} \mathrm{Yb}^{39+}$ permitted injection into the postaccelerator. Accordingly, multiple yrast-band transitions of ${ }^{166} \mathrm{Yb}$ were observed in the CE spectra [see Fig. 1(a)] validated via mutual particle- $\gamma \gamma$ coincidences. The contribution of ${ }^{166} \mathrm{Yb}$ to the beam composition was determined using its known $B(E 2)$ values [28]. Overall, the radioactive ion beam was composed of $82(2) \%{ }^{132} \mathrm{Sn}, 13(1) \%{ }^{132} \mathrm{Sb}, 0.3(1) \%{ }^{132} \mathrm{Ba}$, and $5(2) \%{ }^{166} \mathrm{Yb}$.

To ensure pure electromagnetic excitation, the minimum distance between the nuclear surfaces must exceed $5 \mathrm{fm}$ [29]. Thus, only scattering angles up to $67.6(1)^{\circ}$ in the center-of-mass frame were included in the analysis. Doppler correction was employed for beamlike and targetlike nuclei using kinematic reconstruction. The Dopplercorrected, background-subtracted $\gamma$-ray spectra, which

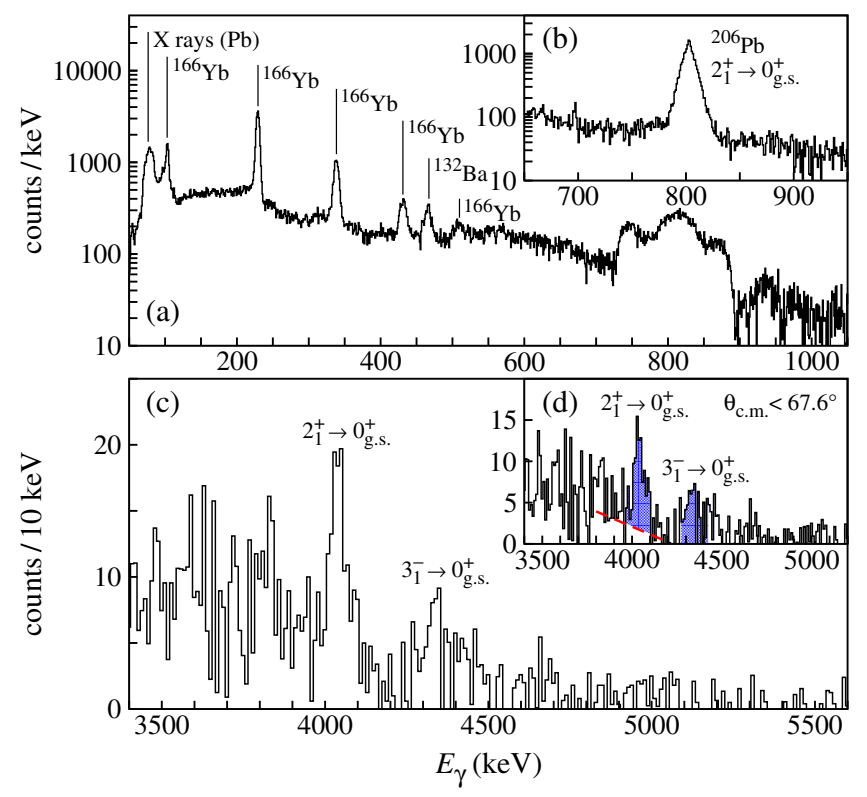

FIG. 1. (a) Low-energy part of the $\gamma$-ray spectrum detected in coincidence with scattered $A=132,166$ nuclei after Doppler correction for nuclei with $A=132$ and background subtraction. Below $600 \mathrm{keV}$, several $\gamma$-ray transitions of the beam contaminants ${ }^{132} \mathrm{Ba}$ and ${ }^{166} \mathrm{Yb}$ are identified. The ground-state transition of ${ }^{206} \mathrm{~Pb}$ is Doppler broadened. (b) Partial spectrum, Doppler corrected for ${ }^{206} \mathrm{~Pb}$. (c) High-energy part of the $\gamma$-ray spectrum. The ${ }^{132} \mathrm{Sn} 2_{1}^{+} \rightarrow 0_{\text {g.s. }}^{+}$and $3_{1}^{-} \rightarrow 0_{\text {g.s. }}^{+}$transitions are identified. Accumulation of counts below $4 \mathrm{MeV}$ is caused by the Compton edge superimposed by statistical fluctuations of $\gamma$ radiation following $\beta$ decay after background correction. (d) Same as in (c) with an additional restriction to scattering angles ensuring pure electromagnetic excitation. The peak integrals used for the analysis are shaded in blue. The red dashed line indicates the background fit. were detected in coincidence to scattered $A=132,166$ particles, are shown in Figs. 1(a)-1(d). The $2_{1}^{+} \rightarrow 0_{\text {g.s. }}^{+}$. transition of ${ }^{206} \mathrm{~Pb}$ appears at $803 \mathrm{keV}$. The $2_{1}^{+} \rightarrow 0_{\mathrm{g} . \mathrm{s} .}^{+}$and $3_{1}^{-} \rightarrow 0_{\mathrm{g} . s .}^{+}$transitions of ${ }^{132} \mathrm{Sn}$ are observed at 4.04 and $4.35 \mathrm{MeV}$, respectively.

The transition intensities of the $2_{1}^{+} \rightarrow 0_{\mathrm{g} . s .}^{+}$and $3_{1}^{-} \rightarrow 0_{\mathrm{g} . s .}^{+}$ deexcitations of ${ }^{132} \mathrm{Sn}$ were determined with relative errors of $22 \%$ and $30 \%$, respectively. For the target excitation of ${ }^{206} \mathrm{~Pb}$, the error is less than $3 \%$. The $3_{1}^{-} \rightarrow 2_{1}^{+}$transition of ${ }^{132} \mathrm{Sn}$ at $310.7 \mathrm{keV}$ was not observed in this work. Its low intensity was expected due to the small branching known from $\beta$-decay studies [27]. Reduced transition probabilities and the respective $\mathrm{CE}$ cross sections of ${ }^{132} \mathrm{Sn}$ were deduced relative to the established $0_{\mathrm{g} . \mathrm{s} .}^{+} \rightarrow 2_{1}^{+}$(E2) cross section of the target nucleus ${ }^{206} \mathrm{~Pb}$ [30] using the coupled-channel code Gosia2 [31,32]. In addition to the measured efficiency-corrected intensities, the detector geometries and the level schemes of ${ }^{132} \mathrm{Sn}$ up to $4.5 \mathrm{MeV}$ and of ${ }^{206} \mathrm{~Pb}$ up to $1.2 \mathrm{MeV}$ were included in the calculations as well as the adopted $B\left(E 2 ; 0_{\text {g.s. }}^{+} \rightarrow 2_{1}^{+}\right)$value of ${ }^{206} \mathrm{~Pb}$ [30] and the abovementioned branching ratio of ${ }^{132} \mathrm{Sn}$. A spherical shape was assumed for ${ }^{132} \mathrm{Sn}$ with zero quadrupole moments. Transition matrix elements were fitted using a least-squares fit. Figure 2 shows a corresponding $\chi^{2}$ surface within the $1 \sigma$ range. The results obtained from GosIA2 provide one global minimum for the set of reduced matrix elements. The final results are summarized in Table I. The present $B(E 2)$ value is consistent with the preliminary results from HRIBF [15-18]. However, the improved energy resolution of the HPGe array and the measured efficiency does allow unambiguous identification of the $2_{1}^{+}$and $3_{1}^{-}$states and their respective excitation cross sections. Moreover, the new transition strengths related to the $3_{1}^{-}$state are compatible with the lower limits deduced from a lifetime measurement [33].

Compared to the neighboring isotopes ${ }^{130,134} \mathrm{Sn}$ [15], the present results confirm the locally enhanced $E 2$ strength in ${ }^{132} \mathrm{Sn}$ predicted by theory [10-12]. This is counterintuitive

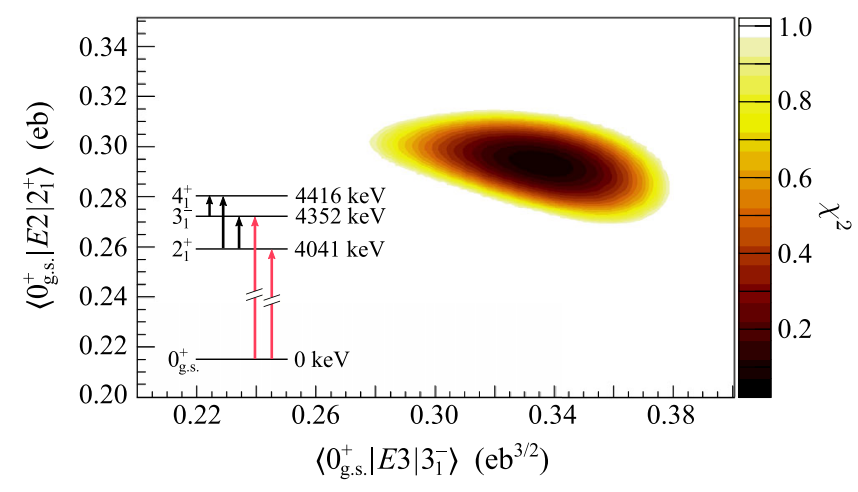

FIG. 2. The $1 \sigma$ contour of the normalized total $\chi^{2}$ surface with respect to the $\left\langle 0_{\text {g.s. }}^{+}|E 2| 2_{1}^{+}\right\rangle$and $\left\langle 0_{\text {g.s. }}^{+}|E 3| 3_{1}^{-}\right\rangle$transitional matrix elements of ${ }^{132} \mathrm{Sn}$ obtained with GosIA2. The inset illustrates the level scheme used for the calculations. 
TABLE I. Reduced transition strengths of ${ }^{132} \mathrm{Sn}$ determined in this work and from previous measurements.

\begin{tabular}{lcccc}
\hline \hline & & \multicolumn{2}{c}{$B\left(E \lambda ; I_{i} \rightarrow I_{f}\right)\left(e^{2} b^{\lambda}\right)$} \\
\cline { 4 - 5 }$I_{i} \rightarrow I_{f}$ & $E_{\gamma}(\mathrm{keV})$ & $E \lambda$ & Present & Previous \\
\hline $0_{\text {g.s. }}^{+} \rightarrow 2_{1}^{+}$ & 4041.2 & $E 2$ & $0.087(19)$ & $0.14(6)[15,16]$ \\
& & & & $0.11(3)[17,18]$ \\
$0_{\text {g.s. }}^{+} \rightarrow 3_{1}^{-}$ & 4351.9 & $E 3$ & $0.11(4)$ & $>0.0512[33]$ \\
$2_{1}^{+} \rightarrow 3_{1}^{-}$ & 310.7 & $E 1$ & $9.1(31) \times 10^{-6}$ & $>3.97 \times 10^{-6}[33]$ \\
\hline \hline
\end{tabular}

to the expected behavior of lowest seniority in doubly magic nuclei. For a microscopic understanding of this feature, state-of-the-art large-scale shell-model (LSSM) and Monte Carlo shell-model (MCSM) calculations have been performed, as well as mean-field calculations utilizing RPA.

The first LSSM calculations for ${ }^{132} \mathrm{Sn}$ are performed in a valence space spanned by the $0 h_{11 / 2}, 1 f_{7 / 2}, 0 h_{9 / 2}, 1 f_{5 / 2}$, $2 p_{3 / 2}, 2 p_{1 / 2}$ orbitals for neutrons and the $0 g_{9 / 2}, 0 g_{7 / 2}$, $1 d_{5 / 2}, 1 d_{3 / 2}, 2 s_{1 / 2}$ orbitals for protons above a closed ${ }^{110} \mathrm{Zr}$ core. This model space allows direct inclusion of $0 \hbar \omega$ quadrupole particle-hole excitations in ${ }^{132} \mathrm{Sn}$. Details on the effective interaction are described in Ref. [34]. The LSSM investigation of ${ }^{132} \mathrm{Sn}$ requires $n p-n h$ excitations from the $\nu 0 h_{11 / 2}$ and $\pi 0 g_{9 / 2}$ orbitals to the valence orbitals across the shell gap to be taken into account. In order to reduce the huge matrix dimension in the $m$ scheme, a truncation scheme was adopted allowing up to $7 p 7 h$ excitations. The Hamiltonian was diagonalized employing the ANTOINE shell-model code [35,36]. Effective charges $e_{\pi}=1.68 e$ and $e_{\nu}=0.41 e$ were used.

A novel MCSM calculation was performed recently in a unified way along the ${ }^{100-138} \mathrm{Sn}$ isotopic chain [37]. A large model space consisting of eight single-particle orbitals for protons and neutrons, i.e., the full $s d g$ harmonic oscillator shell and the $0 h_{11 / 2}, 1 f_{7 / 2}$, and $2 p_{3 / 2}$ orbitals, was used with a fixed Hamiltonian and effective charges $\left(e_{\pi}=1.25 e, e_{\nu}=0.75 e\right)$. Additional information on the $0_{\mathrm{g} . \mathrm{S}}^{+}, 2_{1}^{+}$, and $4_{1}^{+}$states as well as $E 2$ excitation probabilities in ${ }^{132} \mathrm{Sn}$ are deduced employing the same MCSM approach.

Skyrme RPA calculations are performed according to Ref. [38] (cf. also Ref. [9]). The model space is large enough so that appropriate energy-weighted sum rules are well fulfilled: All hole states of ${ }^{132} \mathrm{Sn}$ and particle states up to a maximum energy cutoff of $120 \mathrm{MeV}$ were included, discretized in a spherical box of $20 \mathrm{fm}$. RPA is a proper theory to describe nuclear collective motion. However, while the results for giant resonances only depend on bulk properties of the Skyrme force, those for the low-lying excitations are quite sensitive to the details of the levels close to the Fermi surface. In this respect, measurements are instrumental to test the performance of the Skyrme parameter sets. Additional values derived from a study based on relativistic RPA (RRPA) were taken from Ref. [39].

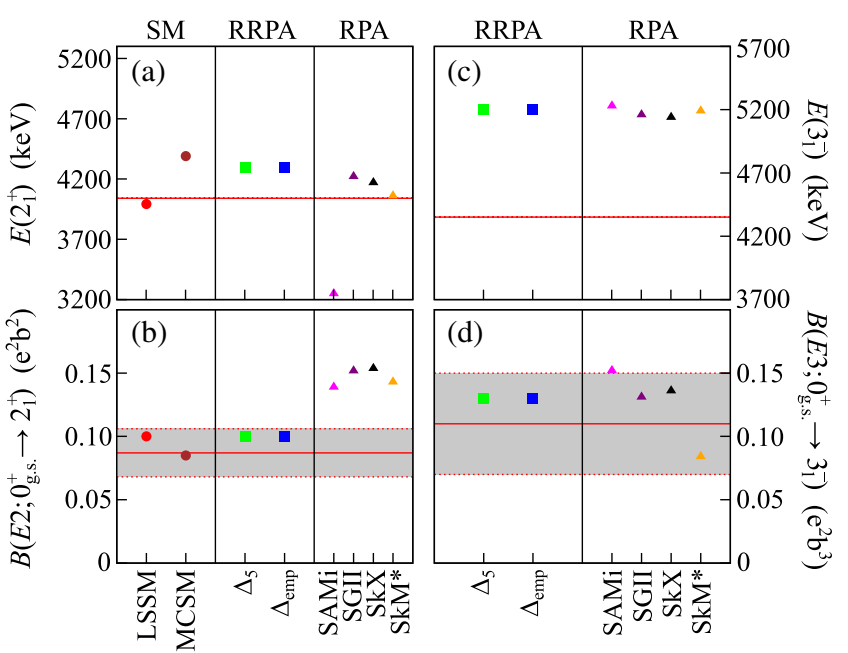

FIG. 3. Comparison of the experimental excitation energies $(\mathrm{a}, \mathrm{c})$ and reduced transition probabilities $(\mathrm{b}, \mathrm{d})$ of ${ }^{132} \mathrm{Sn}$ indicated with red lines, with latest LSSM, MCSM, and mean-field calculations utilizing RPA as well as RRPA (from Ref. [39]) for the $2_{1}^{+}(\mathrm{a}, \mathrm{b})$ and for the $3_{1}^{-}(\mathrm{c}, \mathrm{d})$ state. The gray bands display the $1 \sigma$ deviations of the $B(E \lambda)$ values. Details are given in the text.

The excitation energy of the $2_{1}^{+}$state is well reproduced by most calculations [cf. Fig. 3(a)]. The $B\left(E 2,0_{\mathrm{g} . \mathrm{s} .}^{+} \rightarrow 2_{1}^{+}\right)$ values derived from LSSM, MCSM, and RRPA calculations compare well with the new experimental value within the error bars [cf. Fig. 3(b)]. Both SM approaches corroborate the locally enhanced quadrupole strength in doubly magic ${ }^{132} \mathrm{Sn}$. The calculated $B(E 2)$ values from LSSM yield $0.028,0.100$, and $0.027 e^{2} b^{2}$ for ${ }^{130,132,134} \mathrm{Sn}$, respectively, in agreement with experimental data (cf. Ref. [15] for ${ }^{130,134} \mathrm{Sn}$ ). Corresponding values from MCSM are given in Ref. [37], yielding $0.085 e^{2} b^{2}$ for ${ }^{132} \mathrm{Sn}$. Proton excitations $\pi g_{9 / 2}^{-1} d_{5 / 2}$ across the $Z=50$ shell gap with $\Delta j=\Delta l=2$ are found to be crucial for the evolution of E2 strength along the $\mathrm{Sn}$ isotopic chain. According to the LSSM, these proton excitations amount to a fraction of approximately $14 \%$ of the total wave function of the $2_{1}^{+}$state in ${ }^{132} \mathrm{Sn}$, resulting in an occupation number of 0.21 for the $\pi d_{5 / 2}$ orbital. In neighboring ${ }^{130,134} \mathrm{Sn}$, the occupation is reduced by a factor of about 3. The MCSM result yields a similar trend for the $\mathrm{Sn}$ isotopes with an average $\pi d_{5 / 2}$ occupation of 0.07 for the $2_{1}^{+}$state in ${ }^{132} \mathrm{Sn}$. Although this is not a large number, the contribution to the total E2 matrix element is approximately 25\%. RPA calculations with, e.g., the SkX Skyrme force, yield an expectation value of 0.19 for the proton excitations (cf. Ref. [9]). The $B(E 2)$ value is overestimated by about $60 \%$ [cf. Fig. 3(b)].

Varying structures of the $0_{\mathrm{g} . s .}^{+}$and $2_{1}^{+}$states of $130,132,134 \mathrm{Sn}$ can be visualized by the $T$-plot, as shown in Fig. 4. The MCSM eigenstate is a superposition of $J^{\pi}$ projected MCSM basis vectors [40]. Each basis vector can 


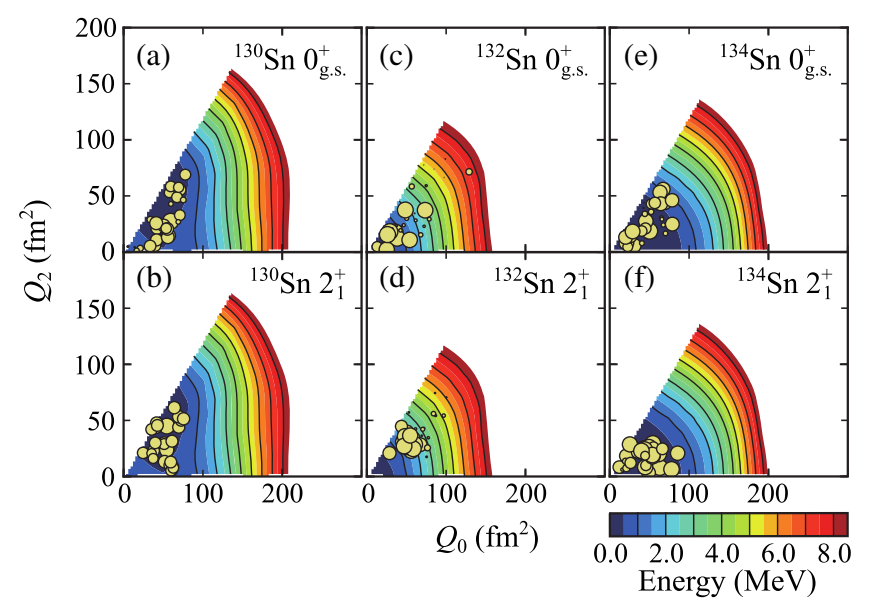

FIG. 4. $\quad T$-plots of the $0_{\text {g.s. }}^{+}(\mathrm{a}, \mathrm{c}, \mathrm{e})$ and $2_{1}^{+}$states $(\mathrm{b}, \mathrm{d}, \mathrm{f})$ of ${ }^{130} \mathrm{Sn}$ $(\mathrm{a}, \mathrm{b}),{ }^{132} \mathrm{Sn}(\mathrm{c}, \mathrm{d})$, and ${ }^{130} \mathrm{Sn}(\mathrm{e}, \mathrm{f})$ show a pronounced potentialenergy minimum for the spherical doubly magic configuration. Circles on the PES represent shape dynamics of MCSM basis vectors. See text for details and discussion.

be characterized by the quadrupole moments $Q_{0}$ and $Q_{2}$, which is plotted as circle on top of the potential energy surface (PES) [41,42]. The area of each circle indicates the overlap probability of the respective MCSM basis vector with the eigenstate. The PES is obtained by constrained Hartree-Fock calculations for the same SM Hamiltonian. Although the PES exhibits a more pronounced spherical minimum for ${ }^{132} \mathrm{Sn}$, the circles are spread outward in the $0_{\text {g.s. }}^{+}$states of ${ }^{130,132,134} \mathrm{Sn}$ shown in Figs. 4(a), 4(c), and 4(e), indicating quantum fluctuations due to pairing correlations (cf. Ref. [41]). However, the major basis vectors of the ground state of ${ }^{132} \mathrm{Sn}$ in Fig. 4(c) are particularly concentrated towards the spherical limit, indicating the well-stabilized double magicity. Moreover, the MCSM calculations provide a high probability of $90 \%$ for the ground state of ${ }^{132} \mathrm{Sn}$ to be in the spherical doubly closed configuration. This value is significantly larger than the corresponding values for the doubly magic isotopes $56,68,78 \mathrm{Ni}$ yielding only $60 \%, 53 \%, 75 \%$ [41]. An average number of $0.08(0.28)$ protons and $0.13(0.22)$ neutrons are excited across the $Z=50$ and $N=82$ shell gaps according to MSCM (LSSM) calculations. For the $2_{1}^{+}$states, the $T$ plots show a notable shift from the near-spherical region of ${ }^{132} \mathrm{Sn}$ [Fig. 4(d)] towards oblate for ${ }^{130} \mathrm{Sn}$ [Fig. 4(b)] but towards prolate for ${ }^{134} \mathrm{Sn}$ [Fig. 4(f)].

Similar to the $B\left(E 2 ; 0_{\mathrm{g} . \mathrm{s} .}^{+} \rightarrow 2_{1}^{+}\right)$, an enhanced octupole transition strength is predicted for the $3_{1}^{-}$state in ${ }^{132} \mathrm{Sn}$ by theory $[11,12]$. Because of the computational limits of the valence space, the SM approaches do not provide information on the $3_{1}^{-}$state. The RPA and RRPA calculations yield $B\left(E 3 ; 0_{\mathrm{g} . \mathrm{s} .}^{+} \rightarrow 3_{1}^{-}\right)$values in agreement with the experimental one within the error bars. However, both calculations overestimate the $E\left(3_{1}^{-}\right)$consistently by about $0.8 \mathrm{MeV}$ [cf. Figs. 3(c) and 3(d)].
Finally, the LSSM and MCSM calculate $E\left(4_{1}^{+}\right)=4.30$ and $4.66 \mathrm{MeV}$, respectively, which is in good agreement with the experimental value of $4.416 \mathrm{MeV}$. Compared to the wave function of the $2_{1}^{+}$state proton excitations, $\pi g_{9 / 2}^{-1} d_{5 / 2}$ are significantly reduced for the $4_{1}^{+}$state, yielding lower average $\pi d_{5 / 2}$ occupation numbers of 0.10 and 0.03 for the LSSM and MCSM calculations, respectively, resulting in $B\left(E 2 ; 2_{1}^{+} \rightarrow 4_{1}^{+}\right)=0.00107 \quad e^{2} b^{2}$ and $0.00219 e^{2} b^{2}$. The latter one compares nicely to the known value of $0.00288(17) e^{2} b^{2}$ [33].

In summary, the new HIE-ISOLDE facility enabled a safe Coulomb-excitation experiment of ${ }^{132} \mathrm{Sn}$ yielding transition strength values for the first two excited states. Novel theoretical approaches allowed detailed insights into the structure of these excitations. For the first time, the doubly magic nucleus ${ }^{132} \mathrm{Sn}$ was explored by MCSM and LSSM calculations. The excitation energies of the $2_{1}^{+}$and $4_{1}^{+}$states and their $B(E 2)$ values were well reproduced. Although a dominant contribution is caused by the $1 p 1 h$ neutron excitation across the $N=82$ shell gap, the contribution of the $\pi g_{9 / 2}^{-1} d_{5 / 2}$ excitation is crucial to reproduce the enhanced E2 strength of ${ }^{132} \mathrm{Sn}$. As the $T$-plots of the $0_{\mathrm{g} . \mathrm{s}}^{+}$. states of ${ }^{130,132,134} \mathrm{Sn}$ exhibited a strong confinement towards the spherical limit, the presented results of experiment and theory can be considered to be the first direct verification of the sphericity and double magicity of ${ }^{132} \mathrm{Sn}$. This is not a trivial fact, as the $N / Z$ ratio differs considerably from unity. Moreover, the MCSM calculations provide a high probability of $90 \%$ for the ground state to be in the doubly closed configuration. For the quadrupole excitation, a shape change from oblate to prolate deformation across the doubly magic configuration was deduced. The $E 3$ transition strengths were well reproduced by the RPA and RRPA results. Future perspectives for experiment and SM theory include challenging measurements of the $B(E 3)$ transition strengths, also of the neighboring isotopes, and their calculation by the extension of the model space. Moreover, numerous experiments at HIE-ISOLDE will investigate nuclei in the vicinity of ${ }^{132} \mathrm{Sn}$ relevant to the astrophysical $r$ process [43].

We thank the HIE-ISOLDE team for the professional support during the experiment. Moreover, we thank M. Zielińska and P. J. Napiorkowski for their support and discussion regarding the GosIA2 analysis. The research leading to these results has received funding from the European Union's Horizon 2020 research and innovation program under Grant Agreement No. 654002. This work was supported by the German BMBF under Contract No. 05P15PKCIA and Verbundprojekt No. 05P2015, in part by the High Performance Computing Infrastructure Strategic Program (Grant No. hp150224), in part by MEXT and Joint Institute for Computational Fundamental Science and a priority issue (elucidation of the fundamental laws and evolution of the universe) to be tackled by using the Post "K" Computer (Grants No. hp160211 and 
No. hp170230), in part by the HPCI system research project (Grant No. hp170182), by the CNS-RIKEN joint project for large-scale nuclear-structure calculations, in part by the Spanish Ministry of Economy, Industry and Competitiveness through Project No. FPA2017-87568-P, by FWO-Vlaanderen (Belgium), by GOA/2010/010 (BOF KU Leuven), and by the Interuniversity Attraction Poles Programme initiated by the Belgian Science Policy Office (BriX network P7/12). A. V. and L. K. thank the Bonn-Cologne Graduate School of Physics and Astronomy for financial support. J. P. and D. M. C. acknowledge the Academy of Finland (Contract No. 265023). G. R. acknowledges support by Bulgarian National Science Fund under Grant No. DN08/23/16. L. P. G. has received funding from the European Union's Horizon 2020 research and innovation program under the Marie SkłodowskaCurie Grant Agreement No. 665779.

"Corresponding author. m.seidlitz@ikp.uni-koeln.de

"Present address: Instituto de Estructura de la Materia, CSIC, Serrano 113bis, E-28006 Madrid, Spain.

[1] H. Grawe, K. Langanke, and G. Martínez-Pinedo, Rep. Prog. Phys. 70, 1525 (2007).

[2] P. A. Evans et al., Science 358, 1565 (2017).

[3] D. Kasen, B. Metzger, J. Barnes, E. Quataert, and E. Ramirez-Ruiz, Nature (London) 551, 80 (2017).

[4] K. L. Jones et al., Nature (London) 465, 454 (2010).

[5] V. Vaquero et al., Phys. Rev. Lett. 118, 202502 (2017).

[6] M. Górska et al., Phys. Lett. B 672, 313 (2009).

[7] J. Taprogge et al., Phys. Rev. Lett. 112, 132501 (2014).

[8] R. L. Kozub et al., Phys. Rev. Lett. 109, 172501 (2012).

[9] G. Bocchi et al., Phys. Lett. B 760, 273 (2016).

[10] J. Terasaki, J. Engel, W. Nazarewicz, and M. Stoitsov, Phys. Rev. C 66, 054313 (2002).

[11] A. Ansari, Phys. Lett. B 623, 37 (2005).

[12] A. Ansari and P. Ring, Phys. Rev. C 74, 054313 (2006).

[13] L. Coraggio, A. Covello, A. Gargano, and N. Itaco, Phys. Rev. C 89, 024319 (2014).

[14] A. P. Severyukhin, V. V. Voronov, I. N. Borzov, N. N. Arsenyev, and N. Van Giai, Phys. Rev. C 90, 044320 (2014).

[15] D. C. Radford et al., Nucl. Phys. A746, 83 (2004).

[16] J. R. Beene et al., Nucl. Phys. A746, 471 (2004).

[17] D. C. Radford et al., Nucl. Phys. A752, 264 (2005).

[18] R. L. Varner et al., Eur. Phys. J. A 25, 391 (2005).
[19] M. J. G. Borge and K. Riisager, Eur. Phys. J. A 52, 334 (2016).

[20] Y. Kadi, Y. Blumenfeld, W. V. Delsolaro, M. A. Fraser, M. Huyse, A. P. Koufidou, J. A. Rodriguez, and F. Wenander, J. Phys. G 44, 084003 (2017).

[21] D. W. Stracener, Nucl. Instrum. Methods Phys. Res., Sect. B 204, 42 (2003).

[22] F. Ames, G. Bollen, P. Delahaye, O. Forstner, G. Huber, O. Kester, K. Reisinger, and P. Schmidt, Nucl. Instrum. Methods Phys. Res., Sect. A 538, 17 (2005).

[23] F. Wenander, J. Instrum. 5, C10004 (2010).

[24] N. Warr et al., Eur. Phys. J. A 49, 40 (2013).

[25] A. N. Ostrowski, S. Cherubini, T. Davinson, D. Groombridge, A. M. Laird, A. Musumarra, A. Ninane, A. di Pietro, A. C. Shotter, and P. J. Woods, Nucl. Instrum. Methods Phys. Res., Sect. A 480, 448 (2002).

[26] J. Eberth et al., Prog. Part. Nucl. Phys. 46, 389 (2001).

[27] Y. Khazov, A. A. Rodionov, S. Sakharov, and B. Singh, Nucl. Data Sheets 104, 497 (2005).

[28] C. M. Baglin, Nucl. Data Sheets 109, 1103 (2008).

[29] D. Cline, Annu. Rev. Nucl. Part. Sci. 36, 683 (1986).

[30] F. G. Kondev, Nucl. Data Sheets 109, 1527 (2008).

[31] T. Czosnyka, D. Cline,, and C. Y. Wu, Bull. Am. Phys. Soc. 28, 745 (1983).

[32] M. Zielińska, L. P. Gaffney, K. Wrzosek-Lipska, E. Clément, T. Grahn, N. Kesteloot, P. Napiorkowski, J. Pakarinen, P. Van Duppen, and N. Warr, Eur. Phys. J. A 52, 99 (2016).

[33] B. Fogelberg, M. Hellström, D. Jerrestam, H. Mach, J. Blomqvist, A. Kerek, L. O. Norlin, and J. P. Omtvedt, Phys. Rev. Lett. 73, 2413 (1994).

[34] H. Naïdja, F. Nowacki,, and K. Sieja, Acta Phys. Pol. B 46, 669 (2015).

[35] E. Caurier, G. Martínez-Pinedo, F. Nowacki, A. Poves,, and A. P. Zuker, Rev. Mod. Phys. 77, 427 (2005).

[36] E. Caurier and F. Nowacki, Acta Phys. Pol. B 30, 705 (1999).

[37] T. Togashi, Y. Tsunoda, T. Otsuka, N. Shimizu, and M. Honma, Phys. Rev. Lett. 121, 062501 (2018).

[38] G. Coló, L. Cao, N. Van Giai, and L. Capelli, Comput. Phys. Commun. 184, 142 (2013).

[39] A. V. Afanasjev and E. Litvinova, Phys. Rev. C 92, 044317 (2015).

[40] T. Togashi, Y. Tsunoda, T. Otsuka, and N. Shimizu, Phys. Rev. Lett. 117, 172502 (2016).

[41] Y. Tsunoda, T. Otsuka, N. Shimizu, M. Honma, and Y. Utsuno, Phys. Rev. C 89, 031301 (2014).

[42] T. Otsuka and Y. Tsunoda, J. Phys. G 43, 024009 (2016).

[43] http://isolde.web.cern.ch/active-experiments. 